\title{
Quantum Physics and Spirituality
}

\author{
Soumyadeep Sarkar \\ Department of Electrical and Electronic Engineering (EEE), Kurukshetra University, Kurukshetra
}

\begin{abstract}
Existence of life, doctrines and theories of science has been at schismatic supremacy since the beginning of life on earth, 3.5 billion years ago. Until recently, the scientific phenomenon known as Quantum Physics has been discovered. And as this theory kicked in to the huge scientific world, it has been widely embraced by many scientific leaders worldwide. The more that is learned about Quantum Physics, the more we discover its Scriptural status and its proof of God's perfect design. So, here's the question which arises; did God really fabricated this design or it was something else, something very unreal which continues to remain a history? Before we come up to a comprehensive theory, let's begin by understanding the core theory of this article, the science of Quantum Physics.
\end{abstract}

Keywords: Quantum mechanics; Einstein- Podolsky- Rosen; consciousness; spiritualism; subatomic science; space time; hyper dimensions; string theory.

\section{A Brief Introduction to Quantum Physics}

\section{What is Quantum Physics?}

Quantum Physics, as we know it, is the most weirdest fundamental branch of Physics ever studied or researched, where we have some bizarre form of concepts like how can something disappear or reappear someplace else, how can we be two places at the same time, but ironically, that's what electrons do all the time. It is the Physics of the subatomic world. Although Quantum Physics is such an outlandish concept but it is also responsible for the technological advances that make modern life possible. Quantum Mechanics gave rise to modern day electronics, cryptography, quantum computing. So basically without Quantum Physics there would be no transistor, and hence no personal computer; no laser absolutely nothing. In essence, Quantum Physics is the study of matter and energy at a nanoscopic scale, beginning within sub atomic particles such as nuclei to atoms and molecules.

Quantum theory also provides accurate descriptions for many previously mysterious and inexplicable phenomena, such as black-body radiation and the stability of the orbital of electrons in atoms.

It has also given realization and recognition into the workings of many different biological systems, including smell receptors and protein structures. Recent studies on photosynthesis have provided evidence that quantum correlations play an essential role in this fundamental process of plants and many other organisms around the planet.

If we tend to shower light on the very history of the beginning of the quantum era, scientific inquiry into the wave nature of light began in the 17th and 18th centuries, when scientists such as Robert Hooke, Christiaan Huygens and Leonhard Euler proposed a wave theory of light based on experimental observations.

In 1803, Thomas Young, an English polymath and physician, performed the famous double-slit experiment that he later described in a paper titled on the nature of light and colours. He demonstrated that light and matter can display characteristics of both traditionally defined waves and particle. Furthermore, it displayed the predominantly probabilistic nature of quantum mechanical phenomena and played a major role in the general acceptance of the wave theory of light.

Considering every single theory of Quantum Physics, what makes these quanta particles so special is that they do not behave in ways according to laws of Physics, making them more of a series of probabilities, rather than something we can scientifically define and observe?

As the human breasts roils for passion for understanding and knowing the actualities hiding behind the quantum theory, a German-born theoretical physicist, very famous and capable, Dr. Albert Einstein came up with a new theory, the theory of relativity.

Einstein is best known in for his famous mass-energy equivalence equation $E=m c^{2}$ (dubbed "the world's most famous equation") bringing him home, the 1921 Nobel Prize in Physics for his "services to theoretical Physics", in particular his discovery of the law of the photoelectric effect, a pivotal step in the evolution of quantum theory.

In 1917, Einstein applied the general theory of relativity to the structure of the universe as a complete conglomeration. He discovered that the general field equations predicted a universe that was dynamic, which is either dilating or diminishing. As observational evidence for a dynamic universe was not testified at the time, Einstein introduced a new term known as the cosmological constant, to the field equations, in order to authorise the theory to predict a static universe. The modified field equations predicted a static universe of closed curvature, in obedience to Einstein's understanding of Mach's principle or Mach's conjecture. Mach's principle is nothing but a hypothesis which suggests that a body's inertial mass results from its interaction with the rest of the matter in the universe. This model became known as the Einstein's World or Einstein's static universe. Einstein's 26 September publication, "Zur Elektrodynamik bewegter Körper" ("On the Electrodynamics of Moving Bodies") harmonized Maxwell's equations for electricity and magnetism with the laws of mechanics, by instigating major changes to the mechanics concentrated to the speed of light. This theory later became known as Einstein's special theory of relativity.

\section{Volume 5 Issue 11, November 2016}




\section{International Journal of Science and Research (IJSR) \\ ISSN (Online): 2319-7064}

Index Copernicus Value (2015): 78.96 | Impact Factor (2015): 6.391

Aftermath of which includes the time-space fabric of a moving body appearing to decelerate and shrivel in the direction of motion, when measured in according to the frame of the observer. Considerably, it was explained that these atoms form molecules and molecules form objects and everything that is seen, is made up of these quanta particle. This paper also proclaimed that the idea of a luminiferous aether, which is also one of the leading theoretical entities in Physics at the time, was superfluous equations. Einstein's 1905 work on relativity remained controversial for many years, but was accepted by leading physicists, starting with Max Planck. Speaking of the space time framework of a moving body, escorted a new theory known as "The Space Time Continuum Theory". Before we shower light on this theory, we must know how Space and Time are correlated to each other.

\section{Understanding Space and Time}

If the most basic definition is considered, a space is nothing but a free space, an expanse which is freely available and not occupied. The definition may appear simple but in terms of science, it is a complete a different new world. Imagine, every single thing in this universe is taken away, the people, the cars and buildings, the earth itself, the planets, the stars and galaxies, not just the big things, also tiny things up to the very last atom, up to the last available evidence of existence of matter; what if they all are taken away? What will remain? When this question is asked, most of us would say 'nothing'. And we would be correct. But incongruously, we would also be wrong. What is actually left behind is an expanse, an empty space. Surprisingly as it turns out, empty space is not actually 'nothing', its 'something', something lot going on inside, something with hidden characteristics, few known to us and few tend to remain a mystery. Space, as it is said, is very real, it is as real as the grain of sand in all the sea beaches, people, planet, stars and everything. In fact, it is so real, that it can bend, it can twist, it can warp, it is so real that it can shape everything in the world all around us and forms the very fabric of the cosmos.

When most of us visualise space or talk about space, the first thing that comes into our mind is the outer space. But, that is not entirely true. The space is everywhere. It is probably the most abundant thing in the universe. Even if we bring this explanation down to the nanoscopic scale, we see atoms. Atoms being the most basic units of matter are entirely made up of $99.9 \%$ empty space. So, ironically, the chair in you are sitting, the television you are watching, the coffee you are drinking, is absolutely made up of nothing, but something. So, we are trying to make sense of something that looks like nothing. Antecedent to the discovery of quantum theory, most scientists believed that space and time only existed in a linear, continuous genre.

And as far as time is considered, as explained by the science of physic, is the indefinite continued progress of existence and events that occur in apparently irreversible succession, advancing from the past through the present to the future. Time is a component and a quantity of various measurements used to sequence events, to compare the duration of events or the intervals between them, and to quantify rates of change of quantities in material reality or in the conscious experience. Time is often referred to as the fourth dimension, along with the three spatial dimensions. Time has long been an important subject of study in religion, philosophy, and science, but defining it in a modus operandi pertinent to all fields without circularity has consistently circumvented the scholars. But what actually is Time? Does time even exists in rudimentary reality?

An explanation was delivered by Dr. Einstein regarding this topic of Time. He said time is flexible, relative and according to Einstein, "the dividing line between past, present, and future is an illusion". So reality is ultimately TIMELESS. This sounds pretty odd from the view of classical Physics, but from the view of consciousness theory and spirituality, it fits in perfectly.

Robert Lawrence Kuhn who is a creator, writer, host and executive producer of the PBS television series "Closer to Truth" interviewed many physicists and philosophers who argued and proclaimed that Time is an illusion, ipso facto believing and accepting the theory of Einstein. Similarly, Huw Price, a professor of philosophy at Cambridge University, argued that the three basic properties of time do not come from the physical world but from our mental states: A present moment that is special; some kind of flow or passage; and an absolute orientation.

"What Physics gives us," Price said, "is the so-called 'block universe,' where time is just part of a four-dimensional space-time and space-time itself is not radical but arise out of some deeper structure."

We sense an "arrow" or direction of time, and even of causation, he said, because our minds add a "subjective ingredient" to reality, "so that we are projecting onto the world the temporal perspective that we have as agents in this environment.

But not all physicists describe time to be an illusion. Nobody actually knows if forever is real because no person has knowledge of a distant event, or the simultaneity of different events, until they are ambivalent in that observer's past. And, therefore, that argument focuses on the fact that the way observers organize their elucidation of the past and cannot manifest the reality of the awaiting future. So opinion highly differs from person to person but many physicists and philosophers now surmise that time is not comprehensive; rather, time arises out of something more fundamental something non temporal, something different, maybe something which is discreet, not continuous and quantized. In the theory of relativity, Einstein explained the face that there may be a difference of elapsed time between two events as measured by the observers either moving relative to each other, or differently placed from a section of a gravitational mass, and termed the phenomena as time dilation. A proof sustaining the fact that time is flexible. The faster we move through space the slower me move through time. He also associated the speed of light in defining the conversion of mater to energy and vice versa.

Now, if I were to describe light in a simple scientific way, I would say, light is nothing but an electromagnetic 


\section{International Journal of Science and Research (IJSR) \\ ISSN (Online): 2319-7064}

Index Copernicus Value (2015): 78.96 | Impact Factor (2015): 6.391

radiation within a certain portion of the electromagnetic spectrum which exists in a tiny packet called photons, which shows duality by presenting properties of both a wave and particle.

In an Euclidean space, the uncoupling between two points is calculated by the distance between the two points. The distance is purely spatial, and is always tends to remain positive. In spacetime, the displacement four-vector $\Delta R$ is given by the space displacement vector (denoted as $\Delta r$ ) and the time difference (denoted as $\Delta t$ ) between the events. The invariant interval, between the two events (in a flat space), $s^{2}$, is elucidated as:

$$
s^{2}=\Delta r^{2}-c^{2} \Delta t^{2}
$$

where ' $c$ ' is the speed of light.

\section{And if presented in terms of time like interval,}

$$
\begin{aligned}
c^{2} \Delta t^{2} & >\Delta r^{2} \\
s^{2} & <0
\end{aligned}
$$

For two events, isolated from each other by a time-like interval, sufficient time drives between them, so, that would result a cause-effect relationship between the two events. For a particle moving through space at less than the speed of light, any two events which may occur or by the particle must be separated by a time-like interval. Event pairs with time-like separation designate a negative spacetime interval $\left(s^{2}<0\right)$ and may be said to occur in each other's future or past. There exists a reference frame such that the two events are observed to prevail in the same spatial location, but there is no reference frame in which the two events can take place, both at the same time. The measure of a time-like spacetime interval is described by the proper time interval $(\Delta \tau)$ :

$$
\Delta \tau=\sqrt{\Delta t^{2}-\frac{\Delta r^{2}}{c^{2}}}
$$

The proper time interval would be measured by the observer himself with a clock proceeding between the two events in an inertial reference frame, when the observer's path decussate each event as that event occurs. The proper time interval technically, defines a real number, since the intramural of the square root is positive.

\section{If explained in terms of light like interval,

$$
\begin{aligned}
c^{2} \Delta t^{2} & =\Delta r^{2} \\
s^{2} & =0
\end{aligned}
$$

In a light-like interval, the spatial separation between two events is exactly balanced by the time linked between the two events. The events define a spacetime interval of zero $\left(s^{2}=0\right)$. Light-like intervals are also known as "null" intervals.

Events which occur to or are commenced by a photon towards its path (i.e., while travelling at ' $c$ ', the speed of light) all have light-like separation. Given one event, all those events which follow at light-like intervals which in turn define the propagation of a light cone, and all the events which preceded from a light-like interval define a second light cone, graphically inverted, which is to say "pastward"

\section{In terms of space like interval,}

$$
\begin{aligned}
c^{2} \Delta t^{2} & <\Delta r^{2} \\
s^{2} & >0
\end{aligned}
$$

When a space-like interval distinguishes two events, sufficient time does not tend to pass between their occurrences for there to generate a causal relationship crossing the spatial distance between the two events at the speed of light or slower. Predominantly, the events are considered not to take place in each other's future or past. There exists a reference frame in such a way that the two events are observed to prevail at the same time, but there is no reference frame in which both the events can occur in the same spatial location.

For these space-like event pairs with a positive spacetime interval $\left(s^{2}>0\right)$, the measurement of space- like separation is the proper distance, $\Delta \sigma$, and the proper distance is given by:

$$
\Delta \sigma=\sqrt{s^{2}}=\sqrt{\Delta r^{2}-c^{2} \Delta t^{2}}
$$

Similarly like the proper time of time-like intervals, the proper distance of space-like spacetime intervals is a real number value and not fractional or something else.

\section{And, if we describe it in the form of interval as an area,}

Time-like or space-like segregations correlate to oppositely oriented rectangles, type of which considered to have rectangles of negative area. The interval has been accorded as the area of an aligned rectangle created by two events and isotropic lines along them. The case of two events separated by light further equates to the rectangle lapsing to the segment between the events and zero area. The transformations generating interval-length invariant are the area-preserving squeeze mappings.

Historically speaking, the parameters which are to be used for the maximum number of times depend on the quadrature of the hyperbola, which is itself, a natural logarithm. This transcendental function is essential in mathematical analysis as its inverse unites circular functions and hyperbolic functions: The exponential function, $\left(\mathrm{e}^{t}, t\right)$ a real number, used in the hyperbola $\left(\mathrm{e}^{t}, \mathrm{e}^{-t}\right)$, generates hyperbolic sectors and the hyperbolic angle parameter. The functions $\cos (\mathrm{h})$ and $\sin (\mathrm{h})$ used with rapidity as provide the common representation of squeeze in the form $\left(\begin{array}{ll}\cosh \phi & \sinh \phi \\ \sinh \phi & \cosh \phi\end{array}\right)$ or in the form of $\boldsymbol{e}^{\boldsymbol{j} \phi}=\cosh \phi+\boldsymbol{j} \sinh \phi$, as the complex unit function.

And, if we study the most fundamental Mathematics of the space time orientation, we find that, the continuum is nothing but a four-dimensional, smooth, connected Lorentzian manifold $(M, g)$, for every physical reason. This means the smooth Lorentz metric $g$ has signature $(3,1)$. The metric governs the geometry of spacetime, as well as establishes the fact that the geodesics of particles and light beams. About each event on this manifold, coordinate charts are used to portray the observers in their own reference time frames. For simplicity's sake, units of measurement are usually chosen such that the speed of light $c$ is equal to 1 . But usually, Cartesian coordinates $(x, y$, $z, t)$ are used.

\section{Volume 5 Issue 11, November 2016}




\section{International Journal of Science and Research (IJSR) \\ ISSN (Online): 2319-7064}

Index Copernicus Value (2015): 78.96 | Impact Factor (2015): 6.391

Identification of the reference frame or observer can be done with one of the coordinate charts; any such observer can describe any event $\boldsymbol{p}$. Similarly, another reference frame may be identified by a second coordinate chart about $\boldsymbol{p}$. As in general, many overlapping coordinate charts are needed to cover a manifold. Provided two coordinate charts, one representing an observer containing $\boldsymbol{p}$ and the other representing the observer as $\boldsymbol{q}$. It is just that the convergence of these charts represents the region of spacetime in which both observers can measure the physical quantities and hence emulate the obtained results. The relation between the two sets of measurements is given by a nonsingular coordinate transformation on this intersection. The proposition of coordinate charts representing local observers who can perform measurements in their proximity also makes a good physical sense of how one can actually accumulate physical information, locally.

Hence, geodesics are elected to be time-like, null, or spacelike if the tangent vector to one point of the geodesic is of this identity. Paths of particles and light beams in a spacetime frame are represented by time-like and null or light-like geodesics, respectively.

Furthermore, space time in general relativity, described by the Minkowski metric $\mathrm{R}^{4}$. This spacetime is named as Minkowski space. The Minkowski metric is usually denoted by $\boldsymbol{\eta}$ and can be written as a four-by-four matrix:

$\eta_{a b}=\operatorname{diag}(1,-1,-1,-1)$

where, the Landau-Lifshitz time-like convention is implemented. Not only this one can also appraise events in Newtonian Physics as a single spacetime. This is GalileanNewtonian relativity, and the coordinate systems are related by Galilean transformations.

However, since these preserve spatial and temporal distances so unconventionally, that particular spacetime can always be disintegrated and fragmented into spatial coordinates plus temporal coordinates, which is scientifically not possible for a general spacetime framework.

To, put this entire thing into a nutshell, I would say spacetime is considered to be continuous, smooth and the mathematical model just combines space and time into single entwined continuum.

And all of these mentioned above brings me to a bafflingly beautiful and at the same time to a very important question. Is God a Mathematician? So, Let us find out.

\section{Is God A Mathematician?}

\section{Mathematics is the language in which god has written the universe. - Galileo Galilei.}

The question of whether god is a mathematician, introduces us to the ostensibly omnipotent powers of Mathematics to outline the world we live in; its "unreasonable effectiveness", a phrase coined by Physics Nobel Laureate Eugene Wigner in 1960. Speaking in the language of a monotheist, as it postulates the belief in the existence of god or in the oneness of God, the god being the Supreme and principal object of faith. So, supposedly if we believe, and stand at the point for atleast once; as from the representations of the bible; the god being the creator of this universe, is he a mathematician? I would say, Yes! He is. And now the most outlandish and bizarre question appears - How? The theology is perfectly answered by Michio Kaku, an American theoretical physicist and futurist. He explains how Mathematics and Physics are correlated to each other.

He says, sometimes Mathematics leads, sometimes Physics leads, sometimes they come together because the use of the Mathematics and Physics altogether in a particular area. For example, in the era of 1600s Sir Isaac Newton, an English physicist and mathematician asked a simple question "If an apple falls, then does the moon also falls?"

Perhaps it's one of the greatest questions ever asked by a member of homo sapiens since the six million of evolution. If an apple falls, does the moon also falls? Isaac Newton says yes. Newton wondered why the Moon doesn't fall .The fact is the Moon is falling ; if it doesn't fall , it would go away from Earth following the line tangent to its orbit .While the Moon travels through the arc, it is falling towards the Earth. So due to the force of gravitation the Moon is continually falling towards the Earth, but it is also continually missing the Earth because it has a tangential velocity and due to inverse square law, so does the apple. He had the unifying theory of the heavens, but he did not have the Mathematics to solve the problem.

So what he did is, he invented calculus. Calculus is is the mathematical study of change, in the same way that geometry is the study of shape and algebra is the study of operations and their application to solving equations. It has two major branches, differential calculus, concerning rates of change and slopes of curves and integral calculus concerning accumulation of quantities and the areas under and between curves.

So calculus is the direct consequence of solving the falling moon problem. Even when we solve calculus for the first time, what we do is we calculate the motion of the falling body. This is exactly how Newton measured and calculated the motion of the falling moon, opening a new world of the celestial mechanics.

So here is a condition where Mathematics and Physics were conjoins like twins, giving birth to a very practical question of calculating the motion of any celestial body.

And then Einstein comes with a different question, he asks, from where the gravity had its origin? Einstein says that gravity is nothing but the aftermath of the curved space. So, why does the apple fall? Why does the moon fall? Why are sitting in the chair and not floating all around in space? Why are we pulled downwards?

Any common people would justify this question by giving the reason of Gravity. But, that is not what Einstein said. He protests that there is no such thing as gravitational attraction. The earth actually warps the space above and around the body, so what the curve does is, it pushes the body to the surface of the planet. . So what Einstein theory meant is gravity does not pull, space pushes to the core of the earth.

\section{Volume 5 Issue 11, November 2016}




\section{International Journal of Science and Research (IJSR) \\ ISSN (Online): 2319-7064}

Index Copernicus Value (2015): 78.96 | Impact Factor (2015): 6.391

So, pushing the very fabric of the reality of space and time requires the differential calculus. So differential calculus is the language of the calculations of the curved surface. So again here is another situation we find, where Mathematics and Physics closely couples up.

But this time Mathematics came first, the theory of curved surface came first. Einstein took that theory of curved surface and then imported it into Physics. So, now we have the String Theory. The String Theory is a theoretical framework in which the point-like particles of particle Physics are replaced by one-dimensional objects called strings. It scientifically describes how these strings propagate through space and interact with each other.

It turns out that a hundred years ago, Mathematics and Physics had to depart from each other. So, when Einstein proposed special theory of relativity in 1905 that was also a around the time, Time Topology came into existence, the topology of the hyper- dimensional objects all around the universe. Spheres, multiverses, multi dimensions, hyper dimensional objects, all came into existence, so Physics and Mathematics had to diverge into different parts. But this time, Maths leaves Physics behind. Mathematics paved its way to the hyperspace and finally mathematicians found expanse where mathematics has no physical applications and mathematics finds itself useless, useless of all is the theory of differential topology in hyper dimensions.

Similarly, with the help of Physics, we figured out the secret of the atomic bomb, we figured out some secrets of the universe and most apparently we discovered the famous String Theory. What is done with String Theory is, we figured out and unlocked the secrets of the Big bang. String Theory propagates us before the big bang, before genesis itself and what does it postulate? It postulates that there exist multiverses of universe itself. So the question is where did the Big Bang come from? How did the Big Bang come into existence? What Einstein says is, we exists in a multiverse of universes, when the collision of two universe takes place, it can form another universe and when an universe splits in half it can form two different universes and that what we think is the Big Bang. The Big Bang is caused either by collision of universes or by fission of the universes.

A String Theory exists in 10 or 11 dimensional hyperspace and these dimensions happen to be super. Not only super, they are super symmetrical. And all of a sudden everyone was shocked, the mathematicians were shocked, the physicists were shocked, and the reason they were shocked is, all of a sudden, Physics gave birth to a new form of mathematics, super numbers, time topology, super differential geometry which further gave birth to a new theory that is the Super symmetric Theory. The Super symmetric theory revolutionised Mathematics.

So, this particular move of Physics, provided equations, which allowed us to unify all the forces of the nature and allowed us to read the mind of the Supreme Soul, it allowed us to read the mind of GOD.

And what is the ultimatum of this equation? The Super Symmetry. And when we try to read this kind of mind, we actually remain being the candidate of reading the mind of God.

The mind holding the entire realm of super symmetry. The mind of god which vibrates like the cosmic music, resonating through an 11 dimensional hyperspace. That is the mind of god. That is the mind of the creator of the fabric of cosmos. The super symmetry that came out of physics, governed by mathematics, which brings me to the final conclusion that, yes! God is a mathematician.

And now, speaking about spirituality, the first thing which comes into our mind is religion. A supernatural realm where God is addressed as the Supreme Being. As far as the science of spirituality is concerned, there has to be some relation between Quantum Physics and Spiritual. So here another question arises, what is the relation between Quantum Physics and spirituality and how they're related.

\section{Association of Quantum Physics With Spirituality}

Spiritualism or Spirituality as it is described is a process of belief or religious practice based on supposed communication with the spirits of the dead, especially through mediums or philosophically speaking, it is the doctrine that the spirit actually co-exists as distinct from matter, or that spirit is the only reality which prevails.

So, what we can say is spiritualism is a metaphysical belief that the world is made up of at least two radical and rudimentary substances that is matter and spirit.

So we understand from Quantum Physics is that, it defines that each and everything coexisting is created due to summation of subatomic sized bits, but the interrogation is what is the that underlying fundamental force holding the quanta particles, atoms and molecules, space and all the other things together? The answer is Electromagnetism. Electromagnetism in the form of photon, that is light. Now what light does is, it keeps electrons fastened to the nuclei of an atom, and which are further bond together with two fundamental force, viz. Strong Force and Electromagnetic force which forges further into forming of molecules, thus objects. All forms of matter are actually made up of this radiating spectrum of electromagnetism, which is Light. The behaviour and characteristics of the electromagnetic radiation depends on its wavelength. Higher frequencies have shorter wavelengths, and lower frequencies have longer wavelengths. When EMR interacts with single atoms and molecules, its functionality depends on the amount of energy per quantum it carries.

Therefore, ameliorating us find our second Biblical evidence of God's representation in the creation of this world, "Then God said, "Let there be light," and there was light. And God saw the light was good. Then he separated the light from the darkness." (Genesis 1: 3-5, New Living Translation) So, what is darkness? Darkness is nothing but the absence of photon particles in the visible wavelength from 400 nanometres to 700 nanometres. As, the bible mentions, "Then he separated the light from the darkness". So was 


\section{International Journal of Science and Research (IJSR) \\ ISSN (Online): 2319-7064}

Index Copernicus Value (2015): 78.96 | Impact Factor (2015): 6.391

god trying to mean space? The "dark" space? Introduction of an electromagnetic spectrum in space?

Throughout the God's approach of the creation of the Genesis, the supreme soul continued creating the grandeur and magnificence of the world, the sky, the planet, the ocean, the sun, the moon, the creatures and finally, the Homo sapiens. Homo sapiens being the only intelligent being having the power of conscience. Light was the first thing that he made before everything else because light was essential to create these things, since his design pattern requires the light force to bind our particles together. Without light nothing would come into existence. Thus, enlightening the facts sustaining the idea of creation of the universe by following specific laws of Quantum Physics.

After god created human beings, they were provided with a specific set of capabilities, which is the ability to think, the ability of being conscious. Human Beings are the only intelligence, who has the highest form of capability to generate conscience. The most conscious being on the planet. As being governed by the laws of quantum physics, gave birth to another question. Is consciousness related to Quantum Physics or it is something supernatural?

\section{Is Consciousness Related to Quantum Physics?}

This question itself is so complex that there exist more than 20,000 papers regarding this topic and none can correctly evaluate it precisely to what it is, ranging from modern neuroscience to philosophy, thinking on the issue showing up in the realm of theology.

When we try to look on something, we try to quantify it; we try to rank it in terms given in the numeric scale, the scale being the unit of measure making it easier for the lay mind to understand. So, at first if we try to understand the situation of an electron in space, the first that comes into the mind of a physicist is the understanding of the planets and its motion. The celestial mechanics of the solar system. Keeping this aside, as we are trying to understand the relation between quantum physics and conscience, there exists a theory by a Japanese- American theoretical physicist, Michio Kaku.

It says that conscience can be divided into three stages. The first stage is the understanding of our position in space. The second stage is the understanding of our position with respect to other people, with respect to motion, hierarchy, politeness and etiquette. And the third explains the ability of evaluating the present and the past to predict the future. Kaku explained something which sort of eludes the philosophers and theologians.

The definition of consciousness, as explained, is the process of equating multiple feedback loops generated by the human brain to create a model of ourselves in space with regard to position and time to satisfy certain goals.

We Homo sapiens are provided with a very special thing in our brain which also differentiates us from other animals, the prefrontal cerebral cortex in our brain, which helps to evaluate the third stage of consciousness. Before we proceed, we plan, we scheme, we try to relate the consequences, but animals don't do this at all. A dog for example, can be taught to do the most complex tricks, but we won't be able to inject the third stage of conscious in his brain, the third stage of consciousness we possess of predicting and evaluating the future.

In another situation, if we try to evaluate consciousness with that of quantum physics, we need to associate it with the Copenhagen Interpretation of Quantum Mechanics.

\section{- Consciousness and the Change of Quantum States by the Process of Observation:}

Consciousness and quantum physics can be explained by associating consciousness with the famous theory of Copenhagen interpretation of Quantum Mechanics. The Copenhagen Interpretation of Quantum Mechanics is an expression of defining the quantum mechanics which was formulated in the years 1925 to 1927 by Niels Bohr and Werner Heisenberg. It remains as one of the most generally taught interpretations of quantum mechanics. So basically what it postulates is that physical systems commonly do not have a definite set of properties before being measured by the observer. So, quantum mechanics can only predefine the probabilities which will ultimately measure and produce certain set of probable results. The procedure of measuring the physical affects the system, causing the set of probabilities to reduce to only one of the possible values immediately after the measurement. This feature is termed as the wave function collapse. So, if we try to measure the physical system the wave function of that particular physical system will collapses due to the consciousness of the observer.

This is that interpretation of quantum mechanics which helped Schrödinger to postulate his experiment on the thought process in the famous "Schrödinger Cat Hypothesis", after he saw as the problem of the Copenhagen interpretation of quantum mechanics applied to everyday objects. This helped him find some sort of absurdity in the thought process of the system. Schrödinger introduced the term Verschränkung which means entanglement while developing the thought experiment. Quantum entanglement is what he meant.

There is also another modified version of this where, John Archibald Wheeler postulates the Participatory Anthropic Principle which says, the entire universe collapsed into the state we see particularly because there had to be some conscious observer present to cause the collapse. Any possible universes which do not contain any conscious observers are automatically not considered.

In the Schrodinger's cat experiment, what happens actually is a cat, a flask of poison; a Geiger counter, a radioactive material, and a hammer were placed inside of a sealed container. The amount of radioactive material so small, that perhaps in the course of the hour one of the atoms decays, but also, with equal probability, that it won't. Now if it is considered to happen, the counter tube discharges and through a relay which releases a hammer that shatters a

\section{Volume 5 Issue 11, November 2016}




\section{International Journal of Science and Research (IJSR) \\ ISSN (Online): 2319-7064}

Index Copernicus Value (2015): 78.96 | Impact Factor (2015): 6.391

small flask of hydrocyanic acid into that sealed box. . If someone leaves this entire system for atleast an hour, one would say that the cat still lives, meanwhile no atom being decayed. The first atomic decay occurs will poison it and the cat dies. The psi-function of the entire system would express this by having the cat neither dead nor alive but the summation of both; alive and dead both at the same time.

Until and unless a person comes and opens the box, it would be dead and alive both at the same time. It would exist in a quantum superposition. But as soon as the person opens it, it observes it and reduces the probability to one, which is either dead or alive

So, if the quantum-mechanical "Schrödinger's cat" paradox is according to the many-worlds interpretation, every event is a branch point. The cat is both alive and dead, regardless of whether the box is opened, but both the "alive" and "dead" cats are in different branches of the universe that are equally real but cannot interact with each other at any hyper dimensional space. Putting these words in between, he also framed a very famous equation, the Schrodinger's wave equation. The Schrödinger equation is the fundamental equation of physics for describing quantum mechanical behaviour of the subatomic particles It is also often called the Schrödinger partial differential wave equation, and is the equation that describes how the wave function of a physical system evolves over time. And it is given by,

$$
\hat{H}|\psi(t)\rangle=i \hbar \frac{\partial}{\partial t}|\psi(t)\rangle
$$

So basically putting the theory in a nutshell, what we do is by opening the box and observing it, we are changing the quantum super positioning state of probability of that particular quantum event to one. Therefore what we see and observe can change the physical outcome of any upcoming event. That is the power, quantum physics has over our consciousness. Another concept of this implementation was given by David Bohm, an American theoretical physicist on the early 1980's.

\section{- David Bohm's Ontological Concept of Quantum} Physics and Human Consciousness:

David Bohm introduced this concept of ontology which is mainly used to describe two different frameworks for understanding the same phenomenon or aspect of the reality. Fundamentally, the concepts were developed in order to explain the bizarre behaviour of subatomic particles, which is usually very difficult to explain by the means of Quantum Mechanics. He stated in his theory that both quantum physics and special theory of relativity were incomplete theories and must converge into some deeper theory. The implicate order, also referred to as the "enfolded" order, is seen as a deeper and more fundamental order of reality. In contrast, the explicate order or "unfolded" order include the speculations that humans normally adjudge. He writes:

"In the enfolded [or implicate] order, space and time are no longer the dominant factors determining the relationships of dependence or independence of different elements. Rather, an entirely different sort of basic connection of elements is possible, from which our ordinary notions of space and time, along with those of separately existent material particles, are abstracted as forms derived from the deeper order. These ordinary notions in fact appear in what is called the "explicate" or "unfolded" order, which is a special and distinguished form contained within the general totality of all the implicate orders"

He advocated the idea that consciousness was somehow the disclosures of this implicate order and that attempting to understand human consciousness and it's relating phenomenal ideas purely by looking at matter in space which has lately preordained and subjected to a massive failure.

However, he could not propose any real scientific mechanism for studying the vast theories of human consciousness and his ontological theory of implicate order never got sufficient propulsion, so this concept never got enlightened to become a fully-prospered theory.

\section{- Roger Penrose: The Theory of Consciousness and The Emperor's New Mind:}

The term consciousness and its understanding emerge from a very complicated computation. So, we have people who do things faster than someone and all the other things. But when we try to receive some information, when we try to learn something, we don't compute, we do something else, something very different. There is something outside the computational laws of physics, which we believe to understand. However the brain as we know it is not a computer. It is a bank containing very sophisticated network of neurons surrounding it.

Roger Penrose, an English mathematical physicist, mathematician and philosopher of science, in his book The Emperor's New Mind: Concerning Computers, Minds, and the Laws of Physics wrote specifically regarding how the laws of physics govern the mind and the consciousness.

Another American cognitive scientist, Marvin Minsky, believed that the brain was a biological computer, a biological machine.

But how the brain is a biological machine? The answer is, unlike any other computer, it rewires itself sequentially when we learn new things. Now as far as this paper is considered, another question arises is does the brain have a soul?

The answer is a bio machine can have a soul. The soul which comprehends information collected by this sophisticated machine. It is still a mystery. But from the view of a physicist, science only believes in something which follows the terms of empirical, testable and demonstratable protocol. So, unless someone finds a way to experimentally provide a solution bound by these terms, it is tough for a physicist to drop to a conclusion.

In Penrose's book, Penrose proclaims that the brain is far more sophisticated than that, perhaps closer to a quantum computer. Specifically, instead of operating on a strictly binary system of "on" and "off," the human brain works with computations that are in a superposition of

\section{Volume 5 Issue 11, November 2016}




\section{International Journal of Science and Research (IJSR) \\ ISSN (Online): 2319-7064}

Index Copernicus Value (2015): 78.96 | Impact Factor (2015): 6.391

different quantum states at the same time. Having the working of the $0 \mathrm{~s}$ and $1 \mathrm{~s}$ both at the same time.

Generally, computers run through programmed algorithms. Penrose delves back into the origins of the computer, by addressing the work of Alan Turing, who developed a device called the "universal Turing machine" which is basically the foundation of the modern computer.

However, Penrose argues that such Turing machine may have some limitations, but that doesn't mean the brain will also have one. Specifically, any algorithmic is constrained by the famous "incompleteness theorem" formulated by Kurt Gödel in the early twentieth century. These systems can never prove their own consistency or inconsistency. However, the human mind can prove some of these results. Therefore, according to Penrose's proclamation, the human mind cannot be an algorithmic system that can be simulated on a computer. We have spent years trying to develop a digital computer which will mimic the brain, but sadistically we couldn't even mimic even $10 \%$ of the functionality of the brain.

The book ultimately presides on a statement that the mind is more than just a brain, which cannot ever be truly simulated within a conventional computer, no matter how complex algorithm the computer possess. In a later book, Penrose proposed together with Stuart Hammer off that the physical mechanism for the quantum physical interactions in the brain are "microtubules" within the brain. Several formulations of how this would work have been disregarded and Hameroff has been told to revise his hypotheses about the exact mechanism. Many neuroscientists (and physicists) have expressed scepticism that microtubules would have this sort of effect.

\section{Determinism, Free Will and Quantum Consciousness:}

As we explained above, how observation by any observer can change the underlying quantum field of that particular event; a detailed but brief explanation of determinism, freewill and quantum consciousness will be followed and understand how they are related to quantum Mechanics.

Now, let me ask you something. Is it that you are reading this entire paper, a decision generated by your own free will, or it was your interest, which was programmed into the universe right from the Genesis? But if free will is an illusion, as many scientists and philosophers have argued, then what happens?

Some supporters of quantum theory and quantum consciousness have framed the idea that quantum indeterminacy is the apparent necessary incompleteness in the description of a physical system, which has become one of the characteristics of the standard description of quantum physics, would mean that quantum consciousness resolves the problem of whether or not humans actually have free will. There's randomness in the quantum world just like the unpredictable sequence of winning numbers on a roulette wheel in any casino in the world. So the statement arises, if our conscious mind works according to the quantum physical processes, then they are not deterministic, and we, therefore, have free will as because a quantum system can never predict an outcome with certainty, it can conclude itself only as a probability from among the various possible states. The values of an observable will be generated non-deterministically in lieu with a probability distribution system which is uniquely determined by the system state itself. It must be noted that the quantum state is destroyed by measurement, so when we refer to a collection of values, each measured value in this collection must be obtained using a freshly prepared state.

There are a number of problems with this, which are summed up in these quotes from neuroscientist Sam Harris in his book Free Will, where he is argues against the theory of free will. He says,

"If certain of my behaviours are truly the result of chance, they should be surprising even to me.How would neurological ambushes of this kind make me free?

The indeterminacy specific to quantum mechanics offers no foothold: If my brain is a quantum computer, the brain of a fly is likely to be a quantum computer, too. Do flies enjoy free will? [...] quantum indeterminacy does nothing to make the concept of free will scientifically intelligible. In the face of any real independence from prior events, every thought and action would seem to merit the statement "I don't know what came over me."

If determinism is true, the future is set -- and this includes all our future states of mind and our subsequent behaviour. And to the extent that the law of cause and effect is subject to indeterminism--quantum or otherwise--we can take no credit for what happens. There is no combination of these truths that seems compatible with the popular notion of free will",

Sam Harris is talking about one of the best known cases of quantum indeterminacy, that is the quantum double slit experiment itself, in which quantum theory tells us that there is absolutely no way to predict with certainty which slit a given particle is going to go through unless we actually make an observation of the particle going through the slit. However, there is nothing about our choice of making this measurement which determines which slit the particle will go through. In the basic configuration of this experiment, there is an even $50 \%$ chance it'll go through either slit, and if we're observing the slits then the experimental results will match that will further distribute randomly. The place in this situation where we can choose whether we're going to make the observation or not.

But that's not the part of the situation that physicists and philosophers are put into, when they're frame working about the quantum indeterminacy, because that is really an option between doing nothing and doing one of two deterministic outcomes each having an outcome of $50 \%$.

\section{Conclusion}

In brief, every theory related to quantum consciousness and spirituality is quite complex.

\section{Volume 5 Issue 11, November 2016}




\section{International Journal of Science and Research (IJSR) \\ ISSN (Online): 2319-7064 \\ Index Copernicus Value (2015): 78.96 | Impact Factor (2015): 6.391}

Hopefully, at some point, there'll be some more interesting scientific evidence on the subject to present, considered the enormous ability of science and physics to explain everything in terms of the sets of protocol, which the science governs itself with.

Finally, to conclude this article, I would say, that if you understand that everything is energy, you also understand that everything you feel, believe and think is also energy. So, your faith, consciousness attitude and focus and those vibrations affect the quantum fields that underly, constitute and determine the outcome of physical matter. So, scientifically your mind has the power to alter the appearance of your physical consequence.

As it is said, Science provided us with theories and equations, which allowed us to unify all the forces of the nature and allowed us to read the mind of GOD.

\section{References}

[1] Spacetime, Available at wikipedia.com

[2] Illusion of time, Available at www.space.com

[3] Schrodinger's equation, available at wikipedia.com

[4] Implicate order and Explicate order by David Bohm, available at Wikipedia.com

[5] Quantum Mechanics, available at www.wikipedia.com

[6] Quantum conscious, Available at www.physics.about.com

[7] The Emperor's new mind, by Roger Penrose.

[8] Quantum theory, by Michio Kaku

[9] Penrose and Hameroff, at www.wikipedia.com

[10] String Theory, available at www.wikipedia.com

[11] Proof of existence, available at www.belief.net

[12] Freewill, available at sciencenews.org.

\section{Author Profile}

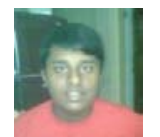

Soumyadeep Sarkar is currently pursuing B. Tech in Electrical and Electronics Engineering from Kurukshetra University, Kurukshetra. He is a research scholar and also an eminent member of The Institute of Electrical and Electronics Engineers (IEEE). His interested areas of research are Electrical Machines and Drives, flexible AC transmission systems, High Voltage AC and Power Electronics. His other research interests include Quantum Physics, Biomedical Science, Classical Mechanics, Astrophysics and Biological Psychiatry. 